\title{
Benign Multicystic Peritoneal Mesothelioma Presenting as Appendiceal Abscess: A Diagnostic and Therapeutic Challenge
}

\author{
Charalampos Seretis ${ }^{\mathrm{a}, \mathrm{b}}$, Ali Mohamed Elhassan ${ }^{\mathrm{a}}$, Leo Kretzmer ${ }^{\mathrm{a}}$, Paul Limª, \\ Anitha Suseelan Menon ${ }^{a}$, Afolabi Awodiya ${ }^{\text {a }}$, Subba Rao Kanchustambam ${ }^{\text {a }}$
}

\begin{abstract}
Primary peritoneal tumors are rarely encountered and their management is usually challenging for the clinicians. Especially when the patients with advanced peritoneal malignancy present as surgical emergencies, usually with symptoms of obstruction, perforation or gross space-occupying lesions, the on-call surgical team has to weigh the pros and cons of urgent versus delayed treatment and plans a safe and simultaneously oncologically beneficial therapeutic approach. Herein, we present a case of a Caucasian man who was referred as suspected complicated appendicitis by his primary care physician, with the final diagnosis being benign multicystic mesothelioma. We describe the challenges of the clinical decision making for the emergency general surgeon and relevant diagnostic and therapeutic pitfalls, which can be potentially minimized by early liaison with tertiary units specializing in the treatment of disseminated peritoneal malignancy.
\end{abstract}

Keywords: Mesothelioma; Appendicitis; Surgery; Laparoscopy; Peritoneum

\section{Introduction}

Peritoneal mesotheliomas are extremely rare primary peritoneal neoplasias, with their incidence estimated at 1:1,000,000 [1, 2]. Having a spectrum of biological behaviors spanning from benign to aggressively malignant, the most frequently presenting symptoms consist of abdominal distension, palpable mass, new onset hernia and gastrointestinal obstruction from extrinsic compression $[3,4]$. Most peritoneal malignancies, prior to the introduction and popularisation of cytoreductive surgery

Manuscript submitted March 17, 2020, accepted April 17, 2020

Published online June 18, 2020

aDepartment of General Surgery, George Eliot Hospital NHS Trust, Nuneaton, Warwickshire, West Midlands, UK

${ }^{b}$ Corresponding Author: Charalampos Seretis, Department of General Surgery, George Eliot Hospital NHS Trust, Nuneaton, Warwickshire, West Midlands CV10 7DJ, UK. Email: babismed@gmail.com

doi: https://doi.org/10.14740/gr1278 and intraperitoneal chemotherapy, were considered an incurable disease, with the mainstay of treatment focusing on symptom control [5].

Due to the rarity of the tumor, diagnostic pitfalls can occur in the diagnostic process, which usually involves as firstline imaging a computed tomography (CT). Therefore, especially when the presenting symptoms are in consistency with intra-abdominal mass, the radiological differential can include tumors from the gastrointestinal tract or intra-abdominal collections $[6,7]$. As a result, frequently the emergency surgical team will come across unexpected intra-operative findings, which will require the transformation of the operative strategy from a standard procedure (for instance radical appendicectomy or segmental colectomy) to an extensive procedure aiming to completely remove all macroscopically visible tumor. The latter becomes a necessity particularly when the patient presents with features of evolving gastrointestinal obstruction or suspected perforation, fact which usually prohibits the adoption of an "open and close" exploratory operation and subsequent referral to a tertiary peritoneal surface malignancy center.

\section{Case Report}

Herein, we present the case of a 61-year-old Caucasian male patient, with no medical comorbidities or prior surgical history, who was referred as suspected acute appendicitis by his general practitioner to our surgical admissions team. His clinical symptoms comprised of an approximately 10-day history of pain in the right iliac fossa without alteration of his bowel habits, rectal bleeding or substantial involuntary weight loss. On admission, clinical examination revealed the presence of localized tenderness in the right iliac fossa, with absence of diffuse peritonism. In addition, a discrete mass was focally palpable in the right iliac fossa, with rectal examination being unremarkable. Baseline blood tests on admission revealed normal white cell and hemoglobin values and essentially normal biochemistry apart from a mild elevation of C-reactive protein (CRP) $(40 \mathrm{mg} / \mathrm{dL}$; reference level 0 - $11 \mathrm{mg} / \mathrm{dL})$. The patient underwent a CT scan of his abdomen and pelvis for further diagnostic assessment. The latter detected the presence of a 17 $\mathrm{cm}$ fluid collection in the right iliac fossa, with another $5 \mathrm{~cm}$ 


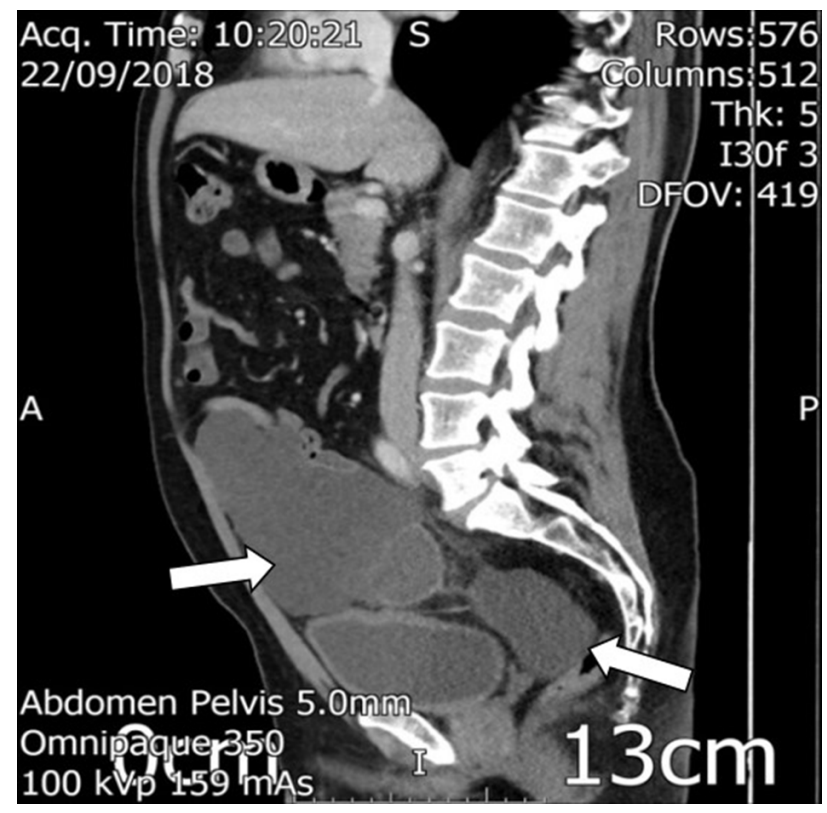

Figure 1. Sagittal view of the right iliac fossa mass and pelvic deposits in the cul-du-sac.

collection located in the pelvis (Figs. 1, 2). Overall, the radiological features were suggestive of an appendiceal abscess and hence the patient was scheduled for a diagnostic laparoscopy with explanation of a greater likelihood of conversion to an open procedure due to the extent of the collections.

Upon entry into the peritoneal cavity, a large gelatinous mass was seen in the right iliac fossa, with nodular deposits in the right parietal peritoneal surface and further gelatinous

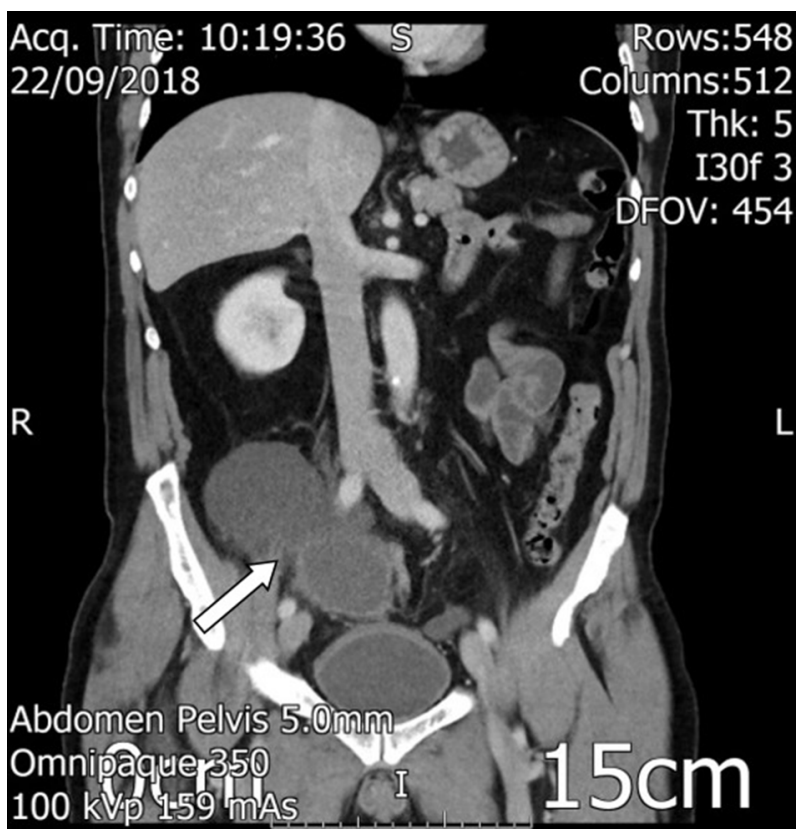

Figure 2. Coronal view of the right iliac fossa mass, demonstarting an enhancing, irregular wall at its inferior aspect, being suggestive of likely appendiceal abscess.

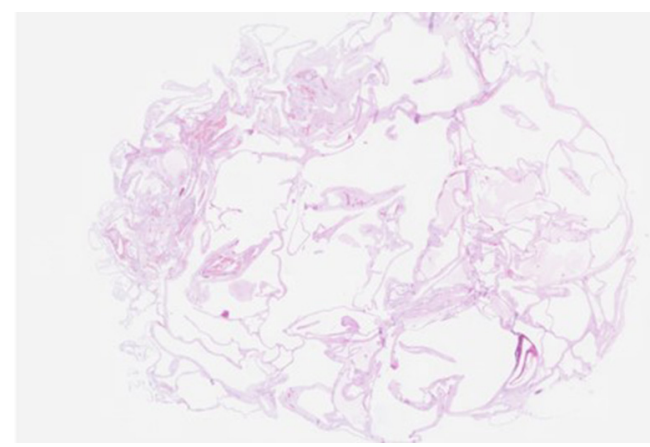

Figure 3. H\&E $\times 5$ magnification: section from the nodular lesion on the serosal aspect of terminal ileum. Low magnification view showing a serosal lesion with multiple cystic spaces of varying sizes. H\&E: hematoxylin and eosin.

cystic deposits in the pelvis. No further deposits were seen elsewhere during thorough laparoscopic assessment. The preliminary diagnosis of a locally advanced, mucinous colonic/ appendiceal tumor with peritoneal metastatic disease was made and after consultation with a second senior colorectal surgeon, the decision was to convert to a midline laparotomy and proceed with right hemicolectomy and maximal tumor debulking. Apart from the right hemicolectomy (enabling en block removal of the right iliac fossa mass), we were able to achieve a complete cytoreduction with performance of infracolic omentectomy, right parietal and pelvic peritonectomy. The patient was transferred to the High Dependency Unit as per routine post emergency laparotomy protocol in our institution and had an uneventful post-operative recovery. He was discharged on the 10th post-operative day and he was further investigated on outpatient basis with a colonoscopy (normal), completion staging CT thorax (no metastases).

Interestingly, the histology was consistent with primary peritoneal benign multicystic mesothelioma, as immuno-histochemistry essays were positive for cytokeratin MNF116, calretinin and negative for CD31 (Figs. 3-7). The above-mentioned features characterized the right iliac fossa multicystic mass, as well as the deposits on the right parietal and pelvic peritoneum and a macroscopically visible cystic deposit on the resected infracolic

Figure 4. H\&E $\times 5$ magnification: representative section from the multicystic lesion present on the serosal aspect of the cecum. H\&E: hematoxylin and eosin. 


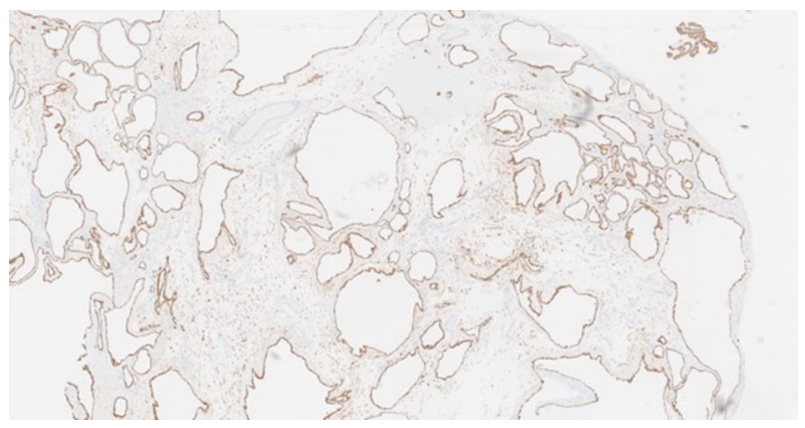

Figure 5. MNF $\times 20$ magnification: cytokeratin MNF116 immunostain highlights the lining cells which are positive.

part of the greater omentum. Taking into account the rarity of the histological findings, the patient's case was discussed in our institution's colorectal multidisciplinary team (MDT) meeting and subsequently it was discussed further in our regional mesothelioma MDT, as well as in the supra-regional peritoneal specialist MDT meeting. The consensus was to evaluate the patient with annual CT scan of his thorax-abdomen-pelvis, as well as tumor markers on periodical basis. The patient was reviewed 6 months after the operation in the outpatient clinic and was updated about the further surveillance pathway.

\section{Discussion}

Benign multicystic mesothelioma represents the most favorable histological subtype of peritoneal mesotheliomas and, despite its name, it is now considered to be a "borderline malignant" rather than a truly benign tumor, characterized by low infiltrative potential but very high rates of loco-regional recurrence after resection [7]. Due to its low infiltrative potential and absence of extra-abdominal metastases, it has the most favorable outcome from all peritoneal mesotheliomas in the series published by high-volume peritoneal malignancy centers, with its treatment consisting of cytoreductive surgery in combination with hyperthermic intraperitoneal chemotherapy $[8,9]$. From those two complimentary modalities though, the key determinant of the long-term outcome is undoubtedly the

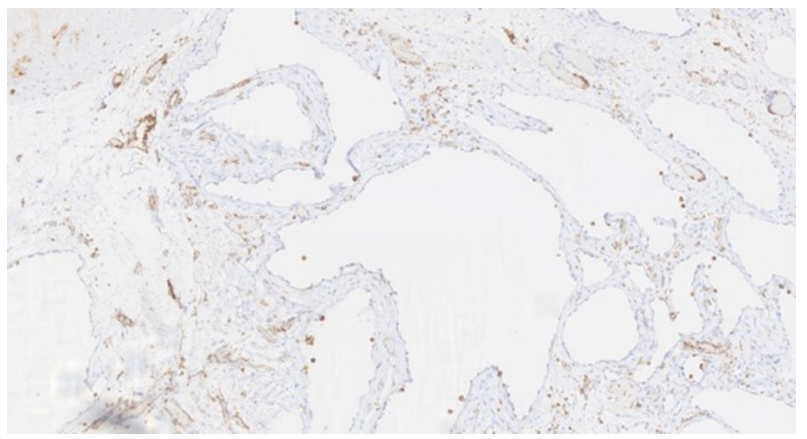

Figure 6. CD31 $\times 100$ magnification: the lining cells are negative for CD31 immunostain indicating that these are not endothelial cells thereby excluding a lymphangioma or a hemangioma. The blood vessels in between the cystic spaces are highlighted.

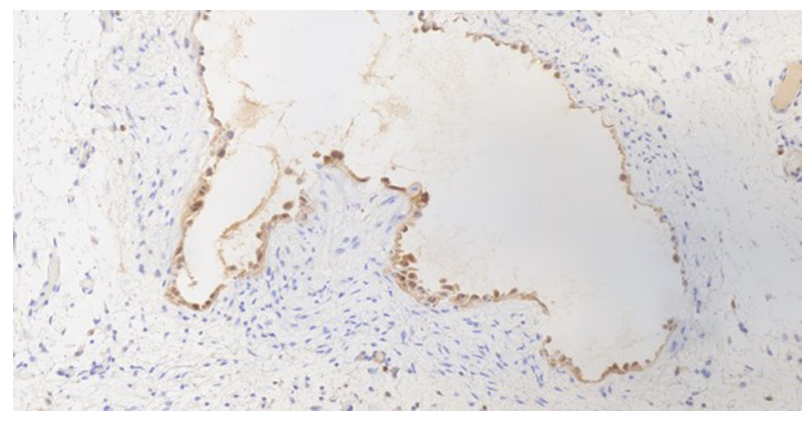

Figure 7. Calretinin $\times 200$ magnification: the lining cells are positive for calretinin immunostain in keeping with mesothelial origin.

successful macroscopic surgical tumor removal and not the administration of intraperitoneal chemotherapy, which only has the auxiliary role of eliminating intraperitoneally floating tumor cells or deposits of millimetric extent. Published data from peritoneal surface malignancies centers indicate that extensive involvement of small bowel serosa, along with presence of high-volume tumor deposits in the upper abdomen, has been associated with greater likelihood of inability to achieve a complete cytoreduction [10]. In our case, the foci of tumor were the right iliac fossa and its ipsilateral parietal peritoneal surface, along with the pelvic peritoneum and on the greater omentum. On the retrospect, the above-mentioned features enabled us to achieve complete macroscopic tumor removal, even in the emergency surgical setting in a non-specialist unit for peritoneal surface malignancies.

Highlighting the learning points from this case, the ability to identify as differential diagnosis the presence of a primary peritoneal malignancy on the pre-operative imaging (overall features were considered as suggestive of appendiceal abscess) could have changed the patient management, as a consult from a specialist tertiary referral center could have been sought. However, upon review of the CT images prior to surgery, the patient was consented appropriately for the possibility of discovering intra-operatively a locally advanced or even disseminated tumor. In addition, the initial performance of a thorough diagnostic laparoscopy and not a percutaneous drainage of the radiologically suspected abscess enabled us to perform a more accurate assessment of the underlying pathology and weighed on the final decision regarding the resectability of the main tumor bulk and its deposits. Should a percutaneous drainage have been attempted, the results would rather complicate the diagnostic pathway due to the presence of mucin and not ascites or purulent effluent. Also, it could result in a risk transfascial implantation of tumor cells unnecessarily, fact which could result in late disease recurrence, which would be extremely difficult to manage and might have required extensive abdominal wall reconstruction with its associated high morbidity.

Summarizing, our case highlights the need for high clinical suspicion of primary peritoneal tumors in patients who present with features of clinically detectable or radiologically suggested multifocal intra-abdominal masses. The latter is of critical importance for correct clinical decision making with respect to operative or non-operative management of the patient. Upon 
doubt, we advocate the early liaison with the reference tertiary referral center for peritoneal surface malignancies in order to obtain appropriate specialist advice. Finally, the appropriate counselling of the patient and relatives is of great importance and should encompass all the potential clinical scenarios.

\section{Acknowledgments}

None to declare.

\section{Financial Disclosure}

None to declare.

\section{Conflict of Interest}

None to declare.

\section{Informed Consent}

We hereby confirm that informed consent was obtained from the patient for publication of the case details.

\section{Author Contributions}

CS, AME, and PL contributed to the literature research and writing of manuscript. LK, ASM, and AA contributed to the editing and reviewing of the manuscript. SRK was the study mentor and contributed to critical revision of the manuscript

\section{Data Availability}

The authors declare that data supporting the findings of this study are available within the article.

\section{References}

1. Mohamed F, Sugarbaker PH. Peritoneal mesothelioma. Curr Treat Options Oncol. 2002;3(5):375-386.

2. Mohamed F, Sethna K, Elias D, Sugarbaker PH. Challenging and unusual cases: Case 3. Peritoneal cystic mesothelioma. J Clin Oncol. 2003;21(7):1419-1420.

3. Feldman AL, Libutti SK, Pingpank JF, Bartlett DL, Beresnev TH, Mavroukakis SM, Steinberg SM, et al. Analysis of factors associated with outcome in patients with malignant peritoneal mesothelioma undergoing surgical debulking and intraperitoneal chemotherapy. J Clin Oncol. 2003;21(24):4560-4567.

4. Acherman YI, Welch LS, Bromley CM, Sugarbaker PH. Clinical presentation of peritoneal mesothelioma. Tumori. 2003;89(3):269-273.

5. Sugarbaker PH. Intraperitoneal chemotherapy and cytoreductive surgery for the prevention and treatment of peritoneal carcinomatosis and sarcomatosis. Semin Surg Oncol. 1998;14(3):254-261.

6. Martin-Jimenez AJ, Aragon-Dominguez V, TrouillhetManso I, Garcia-Gil D. Abdominal mass as a manifestation of mesothelioma. Postgrad Med J. 2018;94(1116):604.

7. Saha A, Mandal PK, Manna A, Khan K, Pal S. Well differentiated papillary mesothelioma of abdomen- a rare case with diagnostic dilemma. J Lab Physicians. 2018;10(2):248-250.

8. Garcia-Fadrique A, Mehta A, Mohamed F, Dayal S, Cecil $\mathrm{T}$, Moran BJ. Clinical presentation, diagnosis, classification and management of peritoneal mesothelioma: a review. J Gastrointest Oncol. 2017;8(5):915-924.

9. Gilani SNS, Mehta A, Garcia-Fadrique A, Rowaiye B, Jenei V, Dayal S, Chandrakumaran K, et al. Outcomes of cytoreductive surgery with hyperthermic intraperitoneal chemotherapy for peritoneal mesothelioma and predictors of survival. Int J Hyperthermia. 2018;34(5):578-584.

10. Chandramohan A, Thrower A, Shah N, Mohamed F. Radiological predictors of complete cytoreduction in 59 patients with peritoneal mesotheliomatreated with cytoreductive surgery and hyperthermic intraperitoneal chemotherapy at a UK referral centre. Colorectal Dis. 2015;17(9):772-778. 